\title{
Increased Autophagy Markers Are Associated with Ductular Reaction during the Development of Cirrhosis
}

Tzu-Min Hung, ${ }^{* \dagger}$ Ray-Hwang Yuan, ${ }^{*}{ }^{\ddagger}$ Wei-Pang Huang, ${ }^{\S}$ Yu-Hsuan Chen, ${ }^{\oplus}$ Yu-Chun Lin, ${ }^{\dagger}$ Chih-Wen Lin,, Hong-Shiee Lai, ${ }^{*}$ and Po-Huang Lee L ** $^{* *}$

From the Department of Surgery, ${ }^{*}$ National Taiwan University Hospital and National Taiwan University College of Medicine, Taipei; the Departments of Medical Research ${ }^{\dagger}$ and Surgery, ${ }^{* *}$ E-DA Hospital, Kaohsiung; the Department of Integrated Diagnostics and Therapeutics, ${ }^{\ddagger}$ National Taiwan University Hospital, Taipei; the Department of Life Science, ${ }^{\S}$ National Taiwan University, Taipei; the Institute of Molecular Medicine, ${ }^{\rrbracket}$ National Taiwan University College of Medicine, Taipei; and the Division of Gastroenterology and Hepatology, "Department of Medicine, E-DA Hospital/I-Shou University, Kaohsiung, Taiwan

Accepted for publication

May 12, 2015.

Address correspondence to Po-Huang Lee, M.D., Ph.D., Department of Surgery, National Taiwan University Hospital, No. 7 Chung-Shan South Rd, Taipei, Taiwan. E-mail: pohuang1115@ntu. edu.tw.

\begin{abstract}
Autophagy is a regulatory pathway in liver fibrosis. We investigated the roles of autophagy in human cirrhotic livers. Cirrhotic and noncirrhotic liver tissues were obtained from patients with hepatocellular carcinoma, and liver tissues from live donors served as control. Patients with cirrhotic livers had significantly increased levels of various essential autophagy-related genes compared with noncirrhotic livers. In addition, colocalization of autophagy marker microtubule-associated protein 1 light chain 3B (LC3B) with lysosome-associated membrane protein-1, increased levels of lysosome-associated membrane protein-2, and increased maturation of lysosomal cathepsin D were observed in cirrhotic livers. By using dualimmunofluorescence staining, we demonstrated that increased LC3B was located mainly in the cytokeratin 19-labeled ductular reaction (DR) in human cirrhotic livers and in an experimental cirrhosis induced by 2acetylaminofluorene (AAF) with carbon tetrachloride $\left(\mathrm{CCl}_{4}\right)$, indicating a conserved response to chronic liver damage. Furthermore, an $\mathrm{AAF} / \mathrm{CCl}_{4}$-mediated increase in $\mathrm{DR}$ and fibrosis were attenuated after chloroquine treatment, suggesting that the autophagy-lysosome pathway was essential for $\mathrm{AAF} / \mathrm{CCl}_{4}$-induced DRfibrosis. In conclusion, we demonstrated that increased autophagy marker positively correlated with DR during the development of cirrhosis. Therefore, targeting autophagy may hold therapeutic value for liver cirrhosis. (Am J Pathol 2015, 185: 2454-2467; http://dx.doi.org/10.1016/j.ajpath.2015.05.010)
\end{abstract}

Autophagy is an intracellular degradation process by which protein aggregates, damaged organelles, and invading microbes are delivered to the lysosome to maintain cellular homeostasis. Surrounding the cytosolic constituents, autophagosome is a double-membrane structure that fuses with a lysosome, forming an autolysosome for subsequent degradation in autophagy. ${ }^{1,2}$ Autophagy was originally discovered from liver experiments. ${ }^{3}$ Features and functions particular to the liver identify it as an organ in which autophagy potentially plays an important role. ${ }^{4}$ Furthermore, increasing evidence indicates that alteration of autophagy is the mechanism behind numerous liver diseases. $^{4-6}$

Cirrhosis, a scarring response that enhances extracellular matrix accumulation after chronic injury, is the last stage of liver fibrosis. It is generally believed that the activation of hepatic stellate cells (HSCs) is a key event in liver fibrogenesis. ${ }^{7}$ Recently, two different groups independently demonstrated that autophagy can regulate lipid droplets in HSCs and then drive HSC activation. ${ }^{8,9}$ HSC activation, both in vitro and in rodent liver injury models, is associated with features of autophagy induction, including a marked increase in autophagic vacuoles, LC3-II levels, and autophagic flux. ${ }^{8,9}$ The blocking of autophagy in cultured cells, with either 3-methyladenine or specific siRNAs to Atg5 or

Supported in part by National Science Council research grants NSC-1012314-B-650-005 (T.M.H.) and NSC-102-2314-B-650-007-MY3 (P.H.L.).

Disclosures: None declared. 
Table 1 Characteristics of the Study Subjects

\begin{tabular}{|c|c|c|c|c|}
\hline Name & Sex & Age, years & Hepatitis & Pathological diagnosis* \\
\hline \multicolumn{5}{|c|}{ Noncirrhotic group } \\
\hline N1 & $\mathrm{F}$ & 59 & No & Donor normal liver \\
\hline $\mathrm{N} 2$ & M & 49 & No & Donor normal liver \\
\hline N3 & M & 78 & $\mathrm{HCV}$ & Metavir score, 1 \\
\hline N4 & M & 40 & $\mathrm{HCV}$ & Metavir score, 0 \\
\hline N5 & M & 58 & $\mathrm{HCV}$ & Metavir score, 1 \\
\hline N6 & M & 63 & $\mathrm{HCV}$ & Metavir score, 2 \\
\hline N7 & M & 68 & $\mathrm{HCV}$ & Metavir score, 2 \\
\hline N8 & M & 64 & $\mathrm{HCV}$ & Metavir score, 1 \\
\hline N9 & $M$ & 79 & HCV & Metavir score, 2 \\
\hline \multicolumn{5}{|c|}{ Cirrhotic group $^{\dagger}$} \\
\hline C1 & $M$ & 70 & $\mathrm{HCV}$ & Grade A \\
\hline C2 & $\mathrm{F}$ & 65 & $\mathrm{HCV}$ & Grade A \\
\hline C3 & M & 53 & $\mathrm{HCV}$ & Grade A \\
\hline C4 & $\mathrm{F}$ & 71 & $\mathrm{HCV}$ & Grade A \\
\hline $\mathrm{C} 5$ & $\mathrm{~F}$ & 74 & $\mathrm{HCV}$ & Grade A \\
\hline C6 & $\mathrm{F}$ & 61 & $\mathrm{HCV}$ & Grade A \\
\hline $\mathrm{C} 7$ & $M$ & 67 & $\mathrm{HCV}$ & Grade B \\
\hline $\mathrm{C} 8$ & $\mathrm{~F}$ & 59 & $\mathrm{HCV}$ & Grade B \\
\hline C9 & $\mathrm{F}$ & 55 & $\mathrm{HCV}$ & Grade C \\
\hline
\end{tabular}

${ }^{*}$ Normal liver had neither serological nor histopathological evidence of liver disease. Metavir score indicates fibrosis from stages 0 to 4: 0 indicates absent, 1 indicates portal fibrosis, 2 indicates portal fibrosis with few septa, 3 indicates septal fibrosis, and 4 indicates cirrhosis.

${ }^{\dagger}$ Cirrhotic liver specimens were obtained from seven patients with hepatitis C-related cirrhosis concurrent with hepatocellular carcinoma (patients $\mathrm{C} 1$ to $\mathrm{C} 7$ ). These patients had Child-Pugh grade A, except patient C7. Two other cirrhotic patients were diagnosed with hepatitis $C$-related cirrhosis for liver transplantation and had Child-Pugh grades $B$ and $C$ (patients $\mathrm{C} 8$ and $\mathrm{C} 9$ ).

F, female; M, male; HCV, hepatitis C virus.

Atg7, leads to attenuated HSC activation and fibrogenesis. ${ }^{8}$ More important, by using a mouse strain with the HSCspecific deletion of $\operatorname{Atg} 7$, Hernández-Gea et $\mathrm{al}^{8}$ demonstrated attenuated fibrosis after sustained liver injury by either carbon tetrachloride $\left(\mathrm{CCl}_{4}\right)$ or thioacetamide. Although these two studies described the role of autophagy in promoting liver fibrosis, the autophagic function in human cirrhotic livers remains largely unknown and requires to be elucidated.

Recently, considerable attention is being directed toward the role of epithelial components (particularly cholangiocytes) in liver fibrosis through the so-called ductular reaction (DR). ${ }^{10}$ The DR, which occurs at the periphery of portal tracts, is a common typical response to injury observed in human liver diseases. ${ }^{11}$ The DR can arise from a proliferation of preexisting bile ductular cells, from hepatic progenitor cells, or from the biliary metaplasia of hepatocytes. The term ductular describes that the containing cells exhibit a ductular phenotype. The term reaction is used to recognize that the epithelial component is accompanied by a complex of extracellular matrix, inflammatory cells, endothelial cells, and mesenchymal cells in the reactive lesions. ${ }^{11,12}$ Increasing evidence indicates a compelling association between the extent of the DR and the severity of fibrosis in patients with chronic hepatitis C (CHC) ${ }^{13,14}$ alcoholic and nonalcoholic fatty liver diseases, ${ }^{15,16}$ and genetic hemochromatosis. ${ }^{17}$

Although the profibrosis effect of autophagy was performed in HSCs, an investigation of autophagy regulation in other liver fibrogenic cells is still lacking. Moreover, clinicopathologic studies to support the link between autophagy and liver fibrosis are insufficient. In this study, both measurements reflecting the number of autophagosomes and measurements of autophagic substrates were applied to patients with and without cirrhosis. We also checked the lysosomal function in these tissue samples. Rats that were treated with 2-acetylaminofluorene (AAF)/ $\mathrm{CCL}_{4}$ were also used as an animal model to confirm the findings in human samples and for a proof-of-concept intervention study.

\section{Materials and Methods}

\section{Human Liver Specimens}

The study design was reviewed and approved by the ethics committee of the National Taiwan University Hospital (Taipei, Taiwan; approval number 201212069RIND). Written informed consent was obtained from each subject. Fourteen liver tissues obtained from patients with $\mathrm{CHC}$ who underwent curative resection for hepatocellular carcinoma (HCC) were classified into two groups: cirrhotic groups (C-group, $\mathrm{C} 1$ to $\mathrm{C} 7$; seven cases) and noncirrhotic group (N-group, N3 to N9; seven cases), according to the pathological examination of the nontumorous region of HCC (Table 1). As controls, two normal liver tissues were obtained from healthy living liver donors and included into the noncirrhotic group (N1 and N2), and two CHCrelated cirrhotic livers from patients receiving liver transplantation were included into the cirrhotic group (C8 and C9). Detailed pathological diagnoses of all 18 subjects are described in Table 1. All subjects were selected randomly, and none of them had received any therapy before surgery. For all subjects, a liver specimen was taken after surgery, immediately snap frozen with isopentane cooled by liquid nitrogen, and stored at $-80^{\circ} \mathrm{C}$ until further use.

\section{Animal Model of Liver Cirrhosis}

Male Sprague-Dawley rats (200 to $250 \mathrm{~g}$ body weight) were raised at $20^{\circ} \mathrm{C}$ to $22^{\circ} \mathrm{C}$ with a 12 -hour light-dark cycle. All animal experimental procedures were approved by the Institute of Animal Care and Use Committees at E-DA Hospital (Kaohsiung, Taiwan). Liver fibrosis was induced by i.p. injection with $50 \%$ of $\mathrm{CCL}_{4}$ (diluted $1: 1$ in olive oil) twice a week for 6 consecutive weeks. To enhance fibrogenic response and increase DR expansion, AAF $(0.02 \%$ in pellet form) was administered for 5 continuous days before the first $\mathrm{CCL}_{4}$ injection and every other day during the period of cirrhosis progression. We 
A
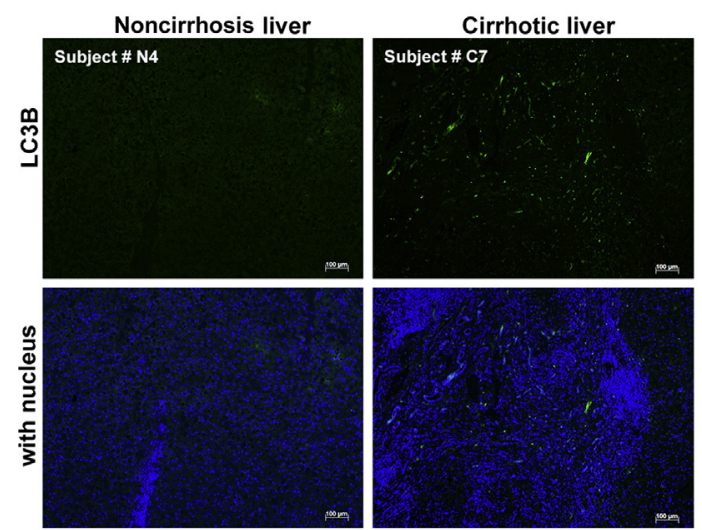

C
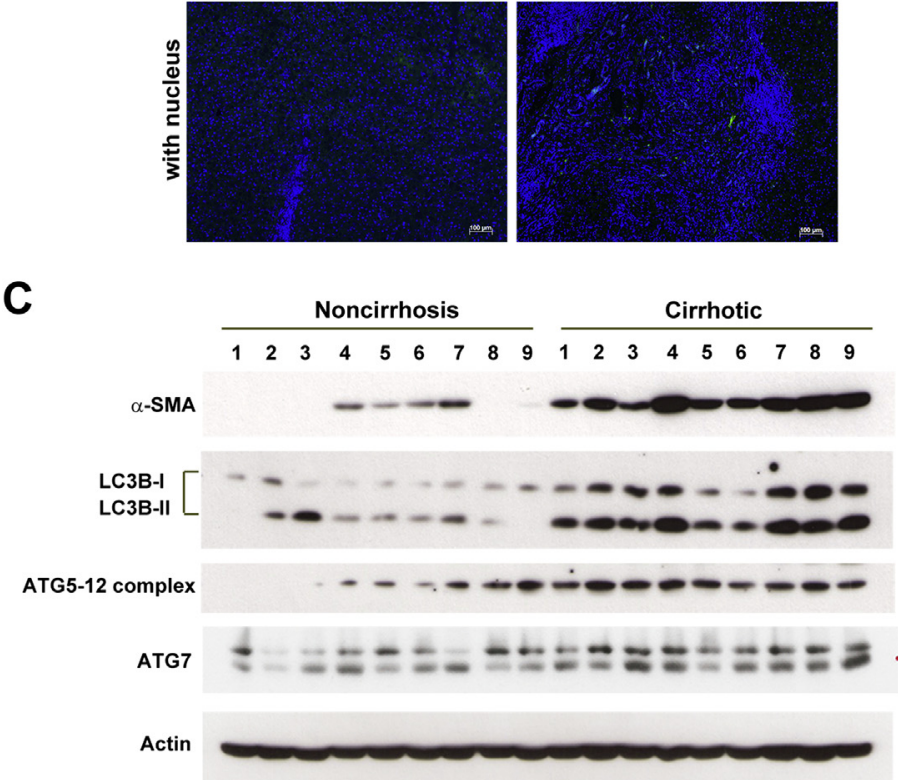

D
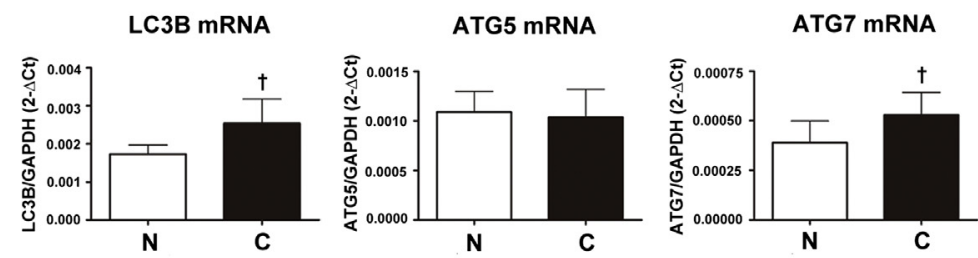

B
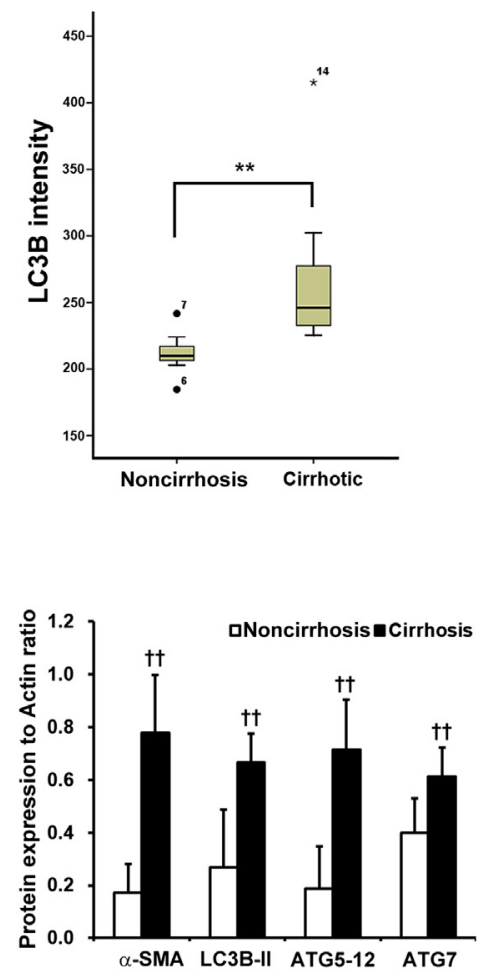

$\mathbf{E}$

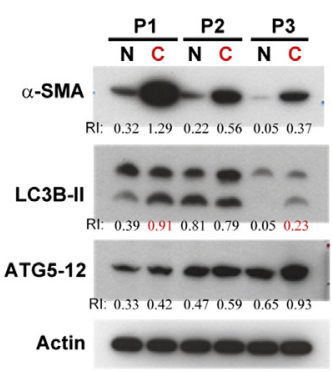

Figure 1 Analysis of autophagy levels in the liver tissues of patients with and without cirrhosis. A: Representative LC3B immunostaining on frozen sections. Note the accumulation of LC3B punctate in cirrhotic liver. B: Fluorescence intensities of LC3B were measured in all 18 subjects and quantified using AxioVision software version 4.8 (Zeiss, Jena, Germany). The numbers 6,7 , and 14 represent outlier subjects. The horizontal line inside the box indicates the median. Significantly increased LC3B levels are present in the cirrhotic group compared with the noncirrhotic group. C: Representative Western blot analysis depicts fibrosis and autophagic markers, including $\alpha$-smooth muscle actin ( $\alpha$-SMA), LC3B, ATG5-12 complex, and ATG7 ( $\beta$-actin used as loading control); the intensity of bands was quantitated using the ImageJ software version 1.49v (NIH, Bethesda, MD; http://imagej.nih.gov/ij), and normalization of $\alpha$-SMA, LC3B, ATG5-12 complex, and ATG7 to $\beta$-actin is shown. D: Quantitative RT-PCR analysis of LC3B, ATG5, and ATG7 in liver tissues obtained from the noncirrhotic (N) and cirrhotic (C) patients. E: Representative Western blot analysis of $\alpha-\mathrm{SMA}, \mathrm{LC} 3 \mathrm{~B}$, and ATG5-12 complex in paired $\mathrm{N}$ and $\mathrm{C}$ tissues of the same patient. The $\mathrm{N}$ specimens were obtained from the first operation for primary hepatocellular carcinoma $(\mathrm{HCC})$ resection. The $\mathrm{C}$ specimens were obtained from the second operation for recurrent HCC resection. Relative intensity (RI) shown was calculated by normalization of the intensities of each marker to the loading control. Data are expressed as means \pm SD from at least three independent experiments (D). $n=9$ for each group (B and $\mathbf{D}) .{ }^{* *} P<0.01, U$ test; ${ }^{\dagger} P<0.05,{ }^{\dagger \dagger} P<$ 0.01 , Student's unpaired $t$-test. GAPDH, glyceraldehyde-3-phosphate dehydrogenase; P1, patient 1; P2, patient 2; P3, patient 3.

used a dose of $2 \mathrm{~mL} / \mathrm{kg}$ for the initial $\mathrm{CCL}_{4}$ injection and 1 $\mathrm{mL} / \mathrm{kg}$ for subsequent injection, as described in the study by Chobert et al. ${ }^{18}$ An additional group of untreated rats was used as a control and fed a standard chow. For measuring autophagic flux, $100 \mathrm{mg} / \mathrm{kg}$ chloroquine (CQ; Sigma, St. Louis, MO) was administered to rats via i.p. injection on the end of 6 weeks $\mathrm{AAF} / \mathrm{CCL}_{4}$ treatment, and the animals were sacrificed 4 hours thereafter. ${ }^{19}$ Rats were injected with phosphate-buffered saline (PBS) as vehicle control. Two independent experiments were performed.

\section{Dual-Immunofluorescence Staining}

Dual-immunofluorescence staining was used to determine the localization of LC3B with lysosome-associated membrane protein-1 (LAMP-1), $\alpha$-smooth muscle actin ( $\alpha$-SMA), and 

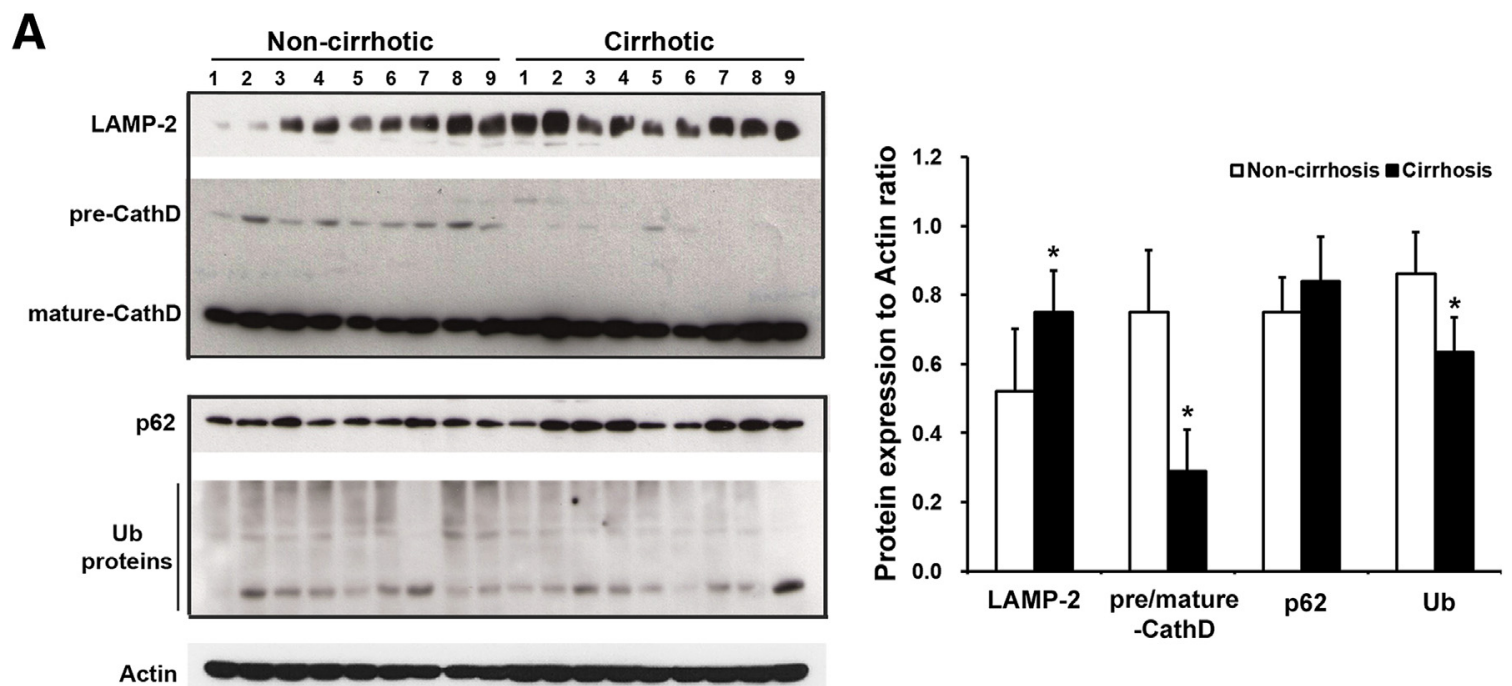

B
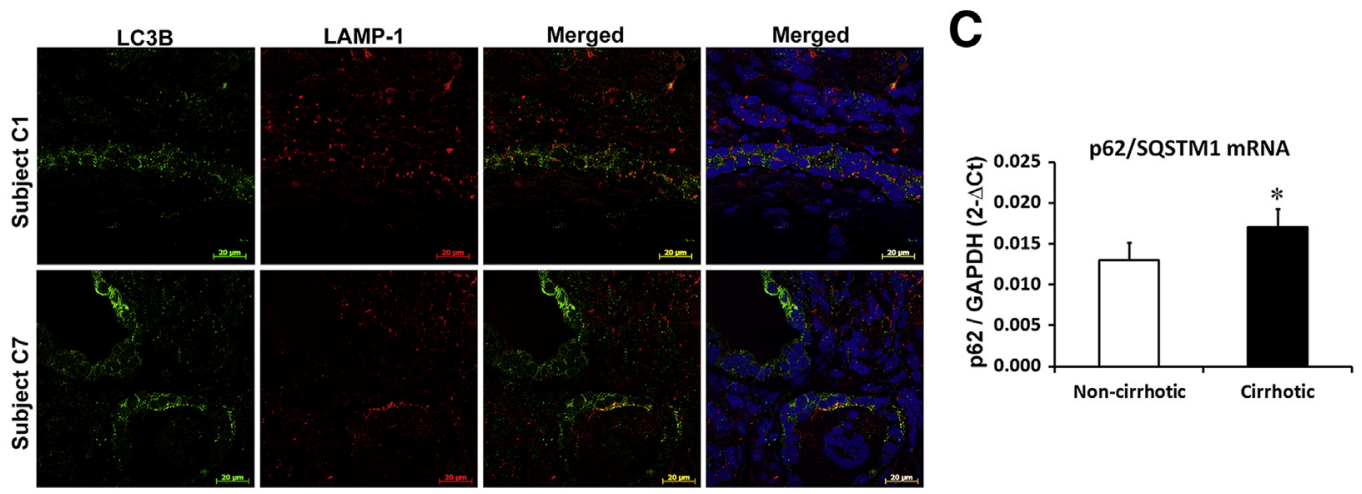

Figure 2 Autophagic functions were assessed in the liver tissues of patients with and without cirrhosis. A: Representative Western blot analysis depicts lysosomal proteins and autophagic substrates, including lysosome-associated membrane protein-1 (LAMP-2), cathepsin D (CathD), p62, and ubiquitinated (Ub) proteins ( $\beta$-actin used as loading control). Note the antibody of cathepsin $D$ can detect both the precursor form (pre-CathD) and the mature form of CathD. The intensity of bands was quantitated using ImageJ software version 1.49v (NIH, Bethesda, MD; $h t t p: / / i m a g e j . n i h . g o v / i j)$, and normalization of LAMP-2, pre-CathD, CathD, p62, and Ub to $\beta$-actin is shown. B: Dual-immunofluorescence staining of LC3B and LAMP-1 in cirrhotic livers. Yellow signals in the merged images indicate the colocalization of these two proteins. C: Quantitative RT-PCR analysis of p62 in liver tissues obtained from the noncirrhotic and cirrhotic patients. Data are expressed as the means \pm SD from at least three independent experiments. $n=9$ for each group. ${ }^{\star} P<0.05$ for noncirrhotic versus cirrhotic (Student's unpaired $t$-test). GAPDH, glyceraldehyde-3-phosphate dehydrogenase.

cytokeratin (CK) 19. Cryosections from liver tissues $(5 \mu \mathrm{m}$ thick) were fixed in $4 \%$ paraformaldehyde in PBS for 10 minutes at $4{ }^{\circ} \mathrm{C}$, and subsequently permeabilized in PBS containing $0.5 \%$ (v/v) Triton X-100 (Sigma) for 15 minutes at room temperature. After incubation with the Image-iT FX signal enhancer (Life Technologies, Carlsbad, CA) and blocking with $20 \%$ normal bovine serum, the sections were incubated with the following primary antibodies: rabbit polyclonal anti-LC3B (dilution, 1:800; Sigma), mouse monoclonal anti-LAMP-1 (dilution, 1:200; BD Bioscience, Franklin Lakes, NJ), anti- $\alpha$-SMA (dilution, 1:500; Sigma), or anti-CK19 (dilution, 1:100; Novocastra, Newcastle-upon-Tyne, UK) antibody at $4^{\circ} \mathrm{C}$ overnight. After washing, the sections were incubated with a cocktail of Alexa Fluor 488-conjugated goat anti-rabbit IgG and Alexa Fluor 594-conjugated goat anti-mouse IgG (dilution, 1:500; Life Technologies) at room temperature for 1 hour, mounted with Vectashield-DAPI (Vector Laboratories, Burlingame, CA), and observed with a Zeiss (Jena, Germany) LSM780 confocal microscope. The negative control condition used nonimmune rabbit $\mathrm{IgG}$ or mouse isotype $\mathrm{IgG}$ instead of the primary antibody.

\section{Western Blot Analysis}

Frozen liver tissues were homogenized, and the total protein extracts were prepared as previously described. ${ }^{20}$ The membranes were probed with the following antibodies: polyclonal anti-LC3B (dilution, 1:4000; Sigma), monoclonal anti-ATG5 (dilution, 1:1000; Sigma), polyclonal antiATG7 (dilution, 1:4000; Sigma), polyclonal anti-LAMP-2 (dilution, 1:2000; GeneTex, Irvine, CA), polyclonal anticathepsin D (dilution, 1:2000; GeneTex), polyclonal antip62/SQSTM1 (dilution, 1:4000; Sigma), monoclonal anti-ubiquitin (dilution, 1:1000; Sigma), and monoclonal anti- $\beta$-actin (dilution, 1:10,000; Novus, Littleton, CO). After overnight incubation with each primary antibody, the 


\section{Cirrhotic liver (Subject C7)}
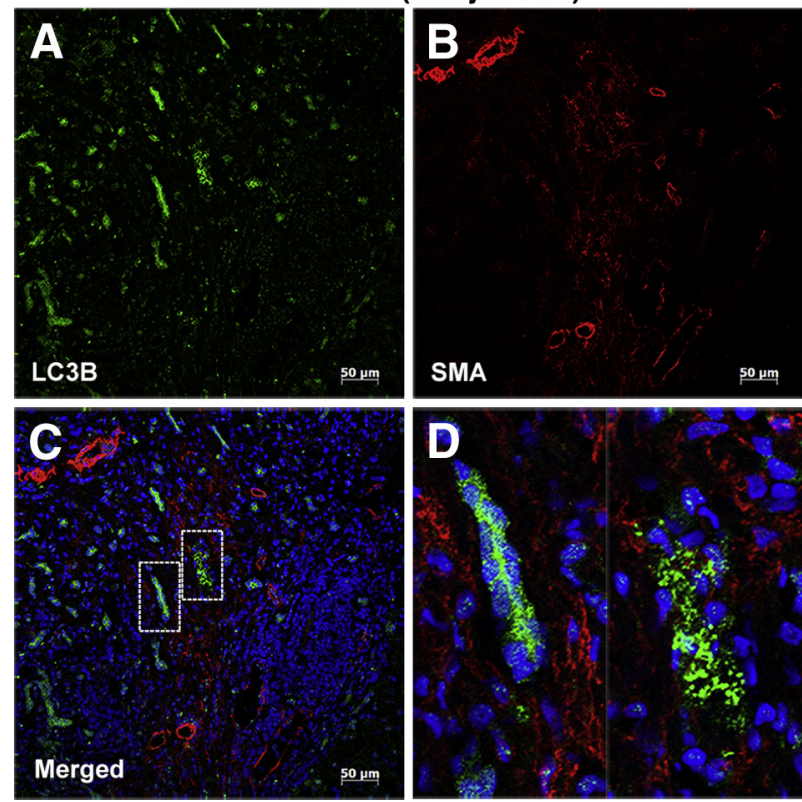

Figure 3 LC3B does not co-express in $\alpha$-smooth muscle actin ( $\alpha$-SMA)positive myofibroblasts. Dual-immunofluorescence staining for $\mathrm{LC} 3 \mathrm{~B}(\mathrm{~A})$ and $\alpha$ SMA (B) in a chronic hepatitis $C$ patient with cirrhosis. Liver tissues were stained with $\alpha$-SMA to identify myofibroblasts (including portal fibroblasts and activated hepatic stellate cells). C: Merged images show no colocalization of LC3B and $\alpha$-SMA. D: Boxed areas in C. A closer look at the ductule structures.

membranes were incubated with horseradish peroxidaseconjugated secondary antibody. The proteins were then detected using an enhanced chemiluminescence detection system (Thermo Fisher Scientific Inc., Waltham, MA).

\section{Isolation of Total RNA and Quantitative Real-Time PCR Analysis}

The total RNA from specimens was extracted using TRIzol reagent (Life Technologies). Total RNA $(1 \mu \mathrm{g})$ was reverse transcribed using random hexamer and Moloney Murine Leukemia Virus Reverse Transcriptase (Thermo Fisher Scientific Inc.). Subsequently, quantitative RT-PCR was performed using TaqMan Gene Expression Assays (Life Technologies). The assay identification numbers of the validated genes are as follows: Hs00797944 for LC3B, Hs00169468 for ATG5, Hs00197348 for ATG7, Hs00177654 for p62/SQSTM1, and Hs99999905 for glyceraldehyde-3-phosphate dehydrogenase. mRNA transcript levels were normalized to glyceraldehyde-3phosphate dehydrogenase mRNA levels $\left(\Delta \mathrm{C}_{\mathrm{T}}\right)$. For profibrogenic gene expression, quantitative RT-PCR was performed using gene-specific primers for collagen type $1 \alpha 1, \alpha$-SMA, and transforming growth factor- $\beta 1$. The primer sequence was the same as our previous study. ${ }^{21}$

\section{Transmission Electron Microscopy}

The specimens were excised and fixed with fixative buffer containing $2 \%$ paraformaldehyde and $2.5 \%$ glutaraldehyde in $0.1 \mathrm{~mol} / \mathrm{L}$ PBS and were stored at $4{ }^{\circ} \mathrm{C}$ until embedding. Tissue samples were then postfixed in $1 \%$ phosphatebuffered osmium tetroxide and embedded in Spurr's resin. Ultrathin sections were stained with $0.2 \%$ lead citrate and $1 \%$ uranyl acetate. Images were acquired using a transmission electron microscope (model TEM-1400; Jeol, Tokyo, Japan).

\section{Statistical Analysis}

All statistical analyses were performed using SPSS version 16.0 statistical software package (SPSS, Chicago, IL). Quantitative variables were presented as the means \pm SD. Between-group comparisons between different groups were performed using the $U$ test or $t$-test. Pearson's correlation was used to determine the correlation coefficients between the expression levels of LC3B and CK19 of liver tissues in 18 patients. All tests were two sided, with a significant $P<0.05$.

\section{Results}

\section{Increased Autophagy Markers in Human Cirrhotic Livers}

Immunofluorescence analysis showed a markedly increased punctate LC3B staining in the cirrhotic livers when compared with noncirrhotic livers (Figure 1A). After quantification, we observed that the intensity of LC3B was significantly higher in the $\mathrm{C}$-group than in the $\mathrm{N}$-group $(P<0.01)$ (Figure 1B).

LC3B-II is a reliable protein marker for autophagosomes, and its levels correlate with the number of autophagic vesicles. To confirm that $\mathrm{LC} 3 \mathrm{~B}$ staining in cirrhotic livers reflects an increase in the autophagosome-bound LC3B-II rather than the cytoplasmic LC3B-I, we used Western blot analysis to investigate the protein expression of LC3B. The LC3B-II protein was expressed abundantly in the cirrhotic livers and only moderately in noncirrhotic livers (Figure 1C). Western blot analysis for other autophagicrelated genes was performed in parallel on all samples in the two groups. The ATG5-12 complex and ATG7 protein levels were significantly higher in the subjects from the Cgroup than in the subjects from the $\mathrm{N}$-group (Figure 1C). The expression of $\alpha$-SMA is used as a fibrogenesis marker.

To establish an overview of the autophagic process, mRNA levels of the autophagy genes were also assessed. In alignment with the protein results, the LC3B and ATG7 mRNA levels were significantly higher in the cirrhotic livers than in the noncirrhotic controls (Figure 1D). To decrease the variability between individual patients, three sets of surgical specimens taken from first operation for the primary HCC and secondary operation for recurrent $\mathrm{HCC}$ in the same patients were used to examine the association between autophagy and cirrhosis. The LC3B-II and ATG5-12 complex protein levels were greater in the cirrhotic tissues compared 
A
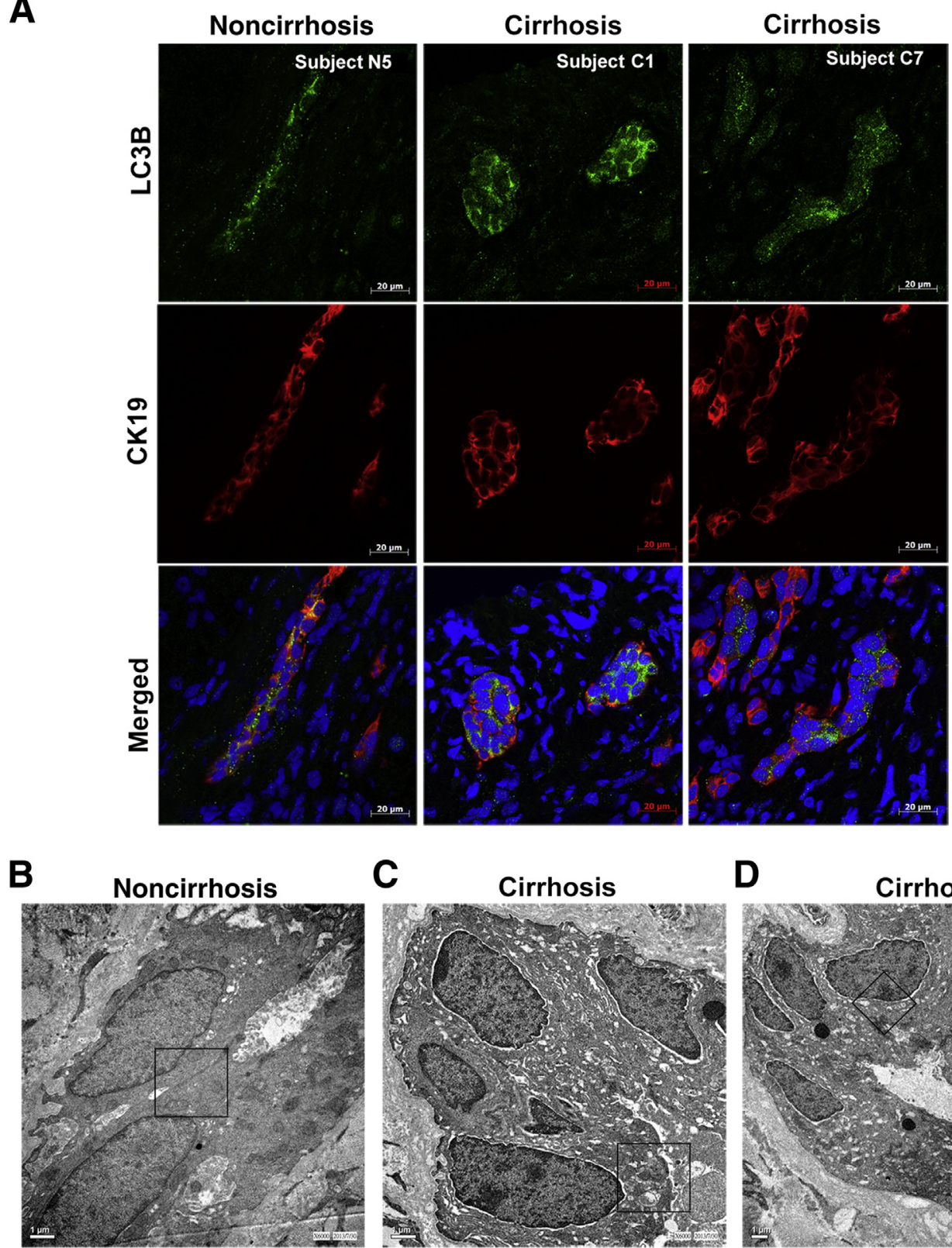

C Cirrhosis

D
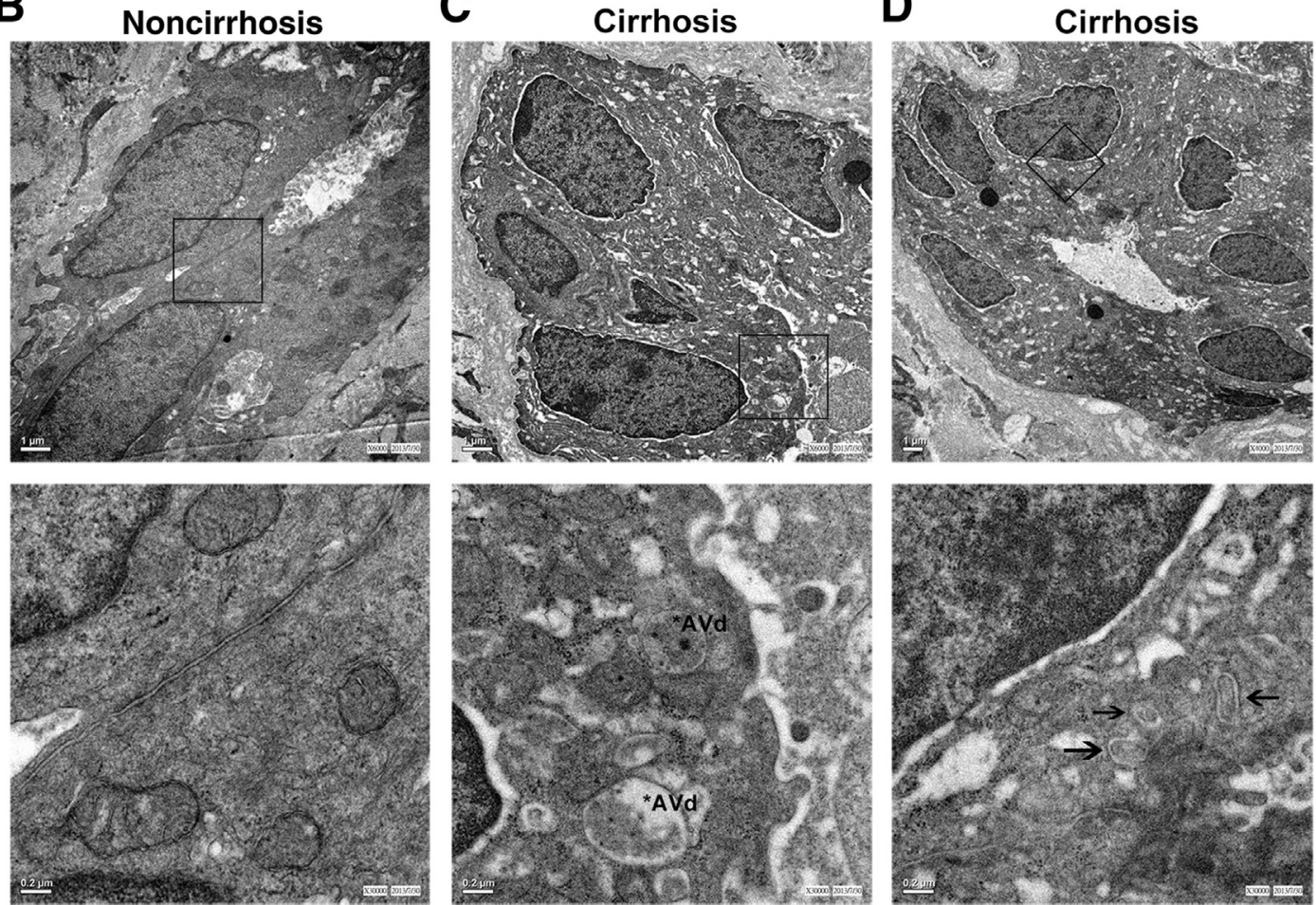

Figure 4 Increased autophagy markers in bile ductular lineage cells. A: LC3B expression in ductular reaction cells. Dual-immunofluorescence images for LC3B (green) and cytokeratin 19 (CK19; red) in three subjects show that CK19-labeled ductular cells express LC3B (merged images). B-D: Ultrastructural assessment of autophagic changes in the bile ductules of livers from a donor and a recipient. Electron micrographs illustrate autophagic vacuoles in the bile ductules from a healthy donor (B) and a cirrhotic recipient (C and D). Higher magnifications of the individual cholangiocyte shown below are enlarged micrographs from the respective boxed area. Arrows point to double-membrane autophagosomes. AVd, degradative autophagic vacuoles. 

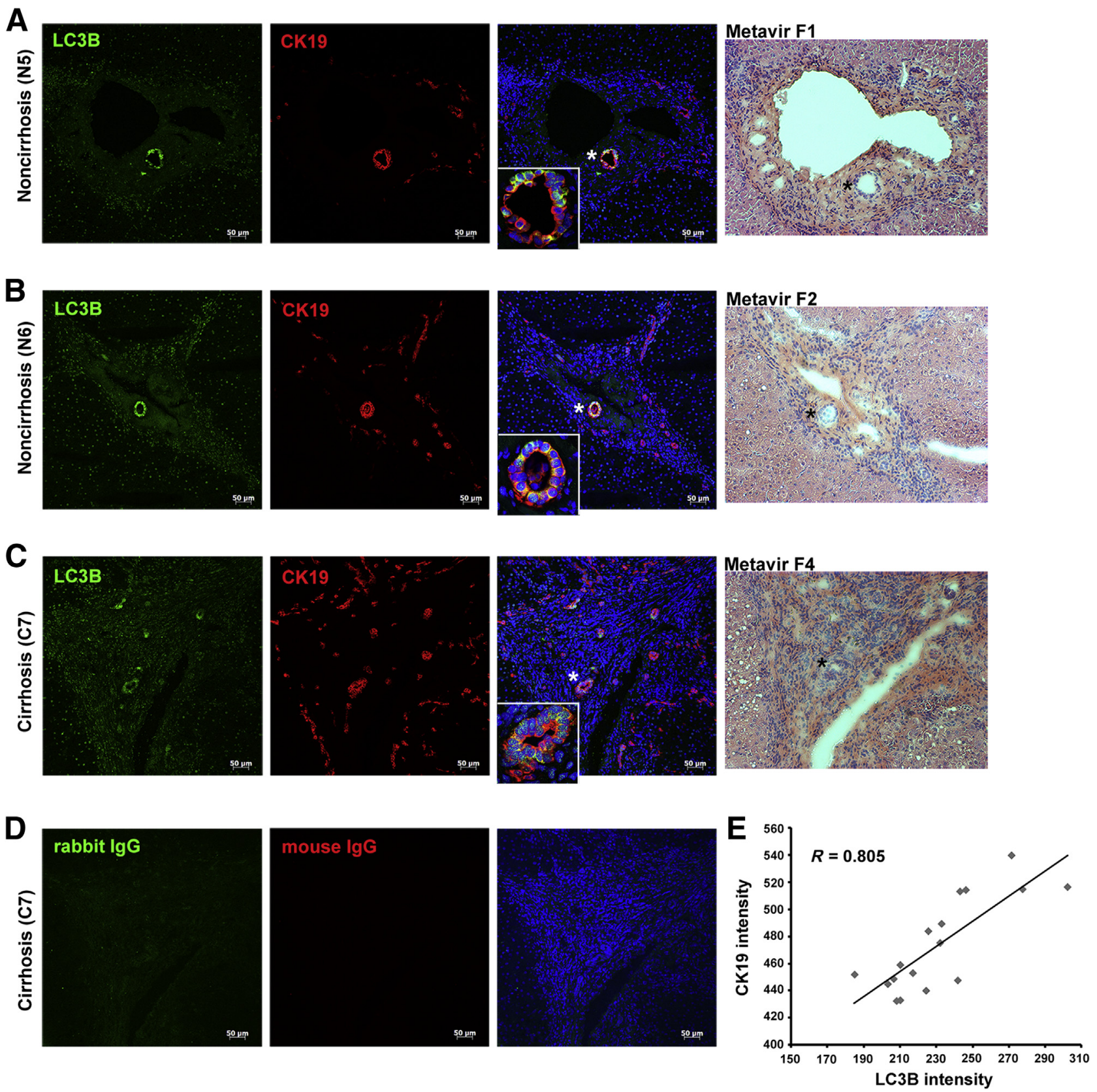

Figure 5 Increased autophagy marker correlates the degree of ductular reaction and fibrosis severity. A-C: Dual-immunofluorescence analysis showing the colocalization of LC3B (green) staining with cytokeratin 19 (CK19; red). The expression of both molecules increased from mild to severe fibrosis. Images are from representative patients with fibrosis stage F1 (A), stage F2 (B), and stage F4 (C). Insets: Higher magnifications of the bile duct (asterisks). D: Negative control counterstain (a consecutive section of the same cirrhotic tissues in which the primary antibodies were replaced with mouse and rabbit normal IgG). E: The fluorescence intensities of the LC3B and CK19 of 18 subjects were measured and used for a correlation analysis with one another. The correlation coefficients $(R)$ and their respective significance levels were calculated using Pearson's correlation analysis. $P<0.001$.

with the noncirrhotic tissues in patients 1 and 3 (Figure 1E). Together, these results demonstrated that increased autophagy markers were present in human cirrhotic livers.

\section{Increased Autophagic Process Could Be Functional in Human Cirrhotic Livers}

Because autophagy is a lysosomal degradation mechanism, lysosomal functions were assessed in parallel in human samples. In cirrhotic livers, the protein levels of LAMP-2 were greater than those in noncirrhotic livers (Figure 2A), suggesting an increase of lysosome number. We next investigated the maturation of the lysosomal hydrolase, cathepsin D. Synthesized as $46-\mathrm{kDa}$ procathepsin $\mathrm{D}$, cathepsin $\mathrm{D}$ is targeted to lysosomes and further cleaved into a mature enzyme comprising noncovalent 28- and 15-kDa polypeptides. By using an antibody that detects both $28-\mathrm{kDa}$ and the precursor forms of cathepsin $\mathrm{D}$, a decrease in the precursor form and an 

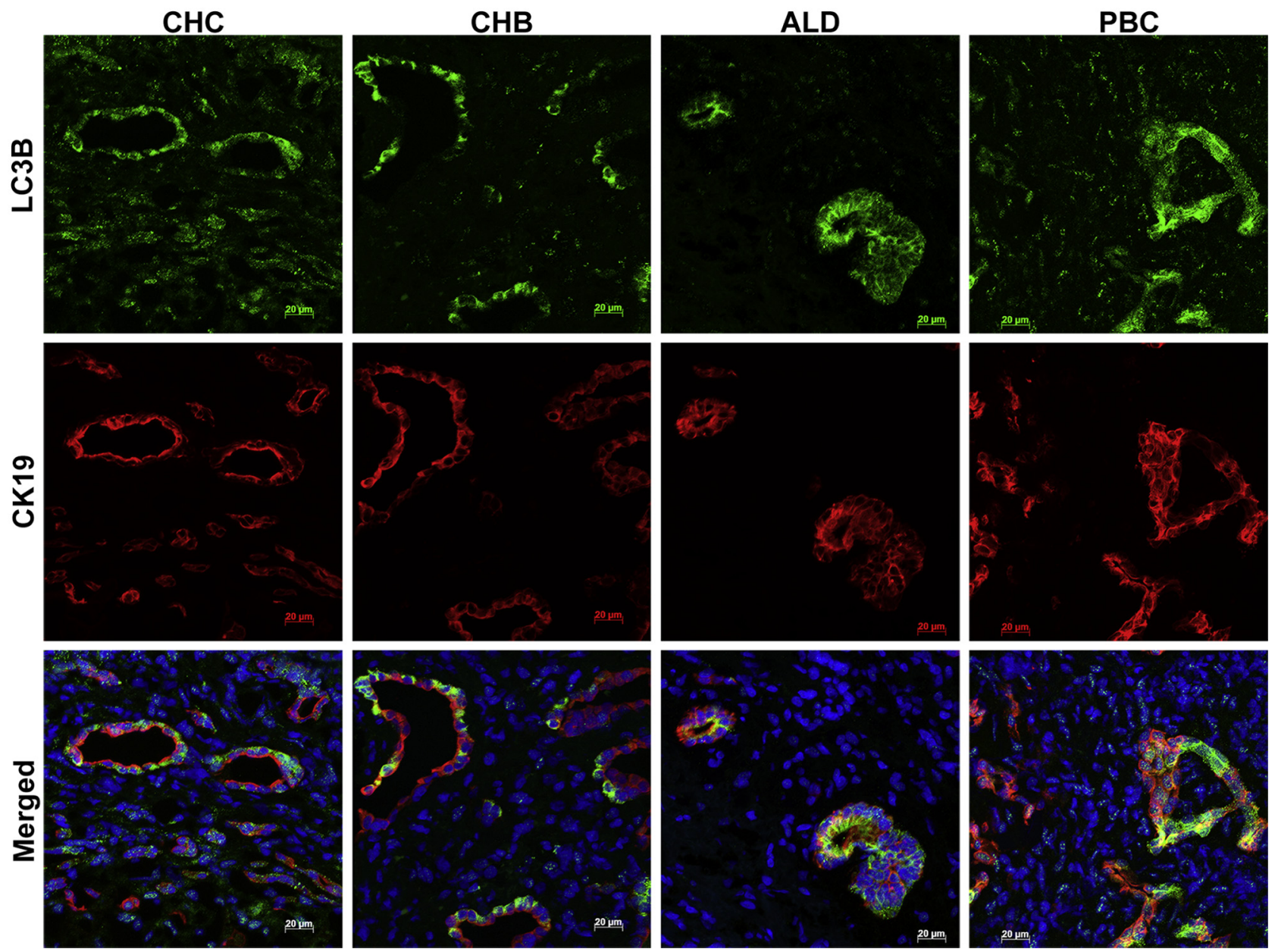

Figure 6 Evidence for increased autophagy marker in ductular reaction in cirrhotic patients with different etiology. Liver sections were obtained from individuals with chronic hepatitis C (CHC), chronic hepatitis B (CHB), alcoholic liver disease (ALD), and primary biliary cirrhosis (PBC), and stained for autophagy marker LC3B and the ductular reaction marker cytokeratin 19 (CK19). All subjects were diagnosed as having liver cirrhosis for liver transplantation, and the cirrhotic specimens were taken during the operation. Dual immunofluorescence for LC3B (green) and CK19 (red) showed that CK19-labeled ductular cells co-express LC3B (yellow in merged images). Representative images of two experiments on liver sections from two patients in each disease etiology.

accompanying increase in the mature 28-kDa form of cathepsin $\mathrm{D}$ were observed in the cirrhotic livers, indicating increased lysosomal activity (Figure 2A).

The clearance of autophagosomes occurs via fusion with lysosomes. We analyzed the subcellular localization of the lysosomal marker LAMP-1 and the autophagosomal marker LC3B using confocal microscopy. The punctate LC3B staining of cirrhotic livers was colocalized with LAMP-1, thereby providing evidence of the fusion of autophagosomal compartments with lysosome (Figure 2B).

To examine the autophagic flux in another manner, we analyzed the levels of the LC3-binding protein p62, which is known to be degraded by autophagy and to act as an adaptor to convey ubiquitinated proteins to autophagosomes for lysosomal degradation. ${ }^{22}$ There was no significant difference in the protein levels of p62 between the noncirrhotic and cirrhotic livers (Figure 2A). However, a decrease in the ubiquitinated proteins was observed in the cirrhotic livers (Figure 2A). It has been reported that increases in the amount of p62 are seen in some situations where there is an increase in autophagic flux, and they might be explained by simultaneous transcriptional induction of the gene encoding p62. ${ }^{23,24}$ Quantitative RT-PCR analysis showed the p62 mRNA levels to be significantly higher in the cirrhotic livers than in the noncirrhotic controls (Figure 2C), further supporting this possibility.

Increased Autophagy Marker Correlates the Degree of DR and Fibrosis Severity

We performed confocal colocalization studies to further identify which cells express abundant LC3B in cirrhotic livers. We initially speculated that the previously reported release of lipids through autophagy could be the mechanism underlying the association between the activation of HSCs and liver fibrogenesis. ${ }^{8}$ However, we did not observe LC3B to be colocalized with $\alpha$-SMA, a marker of activated HSCs (Figure 3). In contrast, LC3B staining was strongly positive 

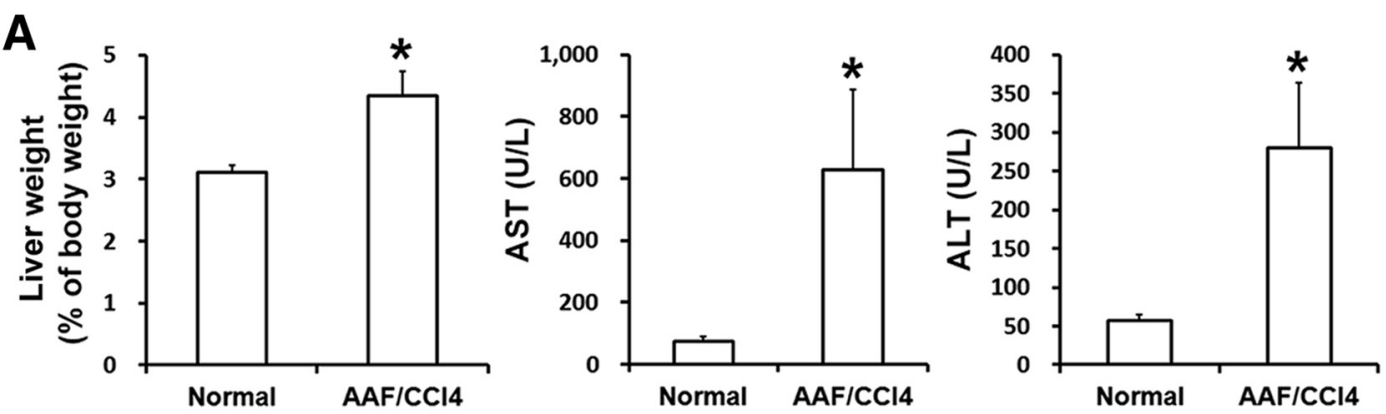

B
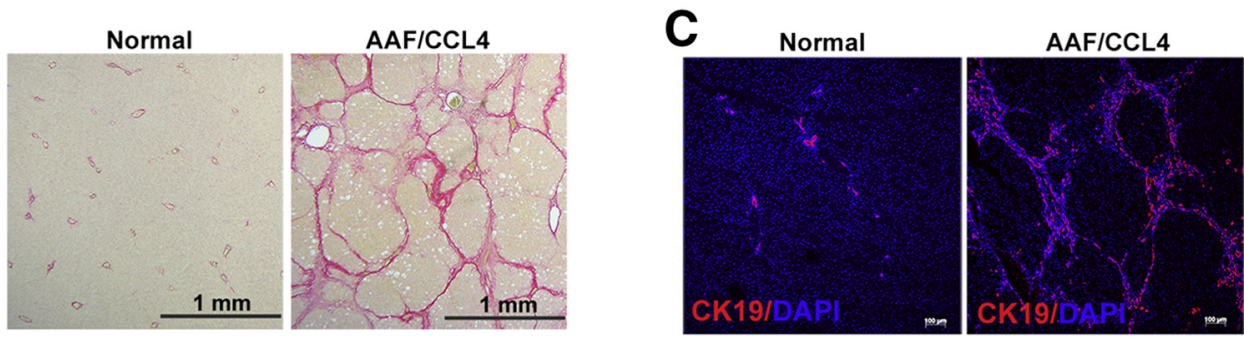

D Normal

AAF/CCL4
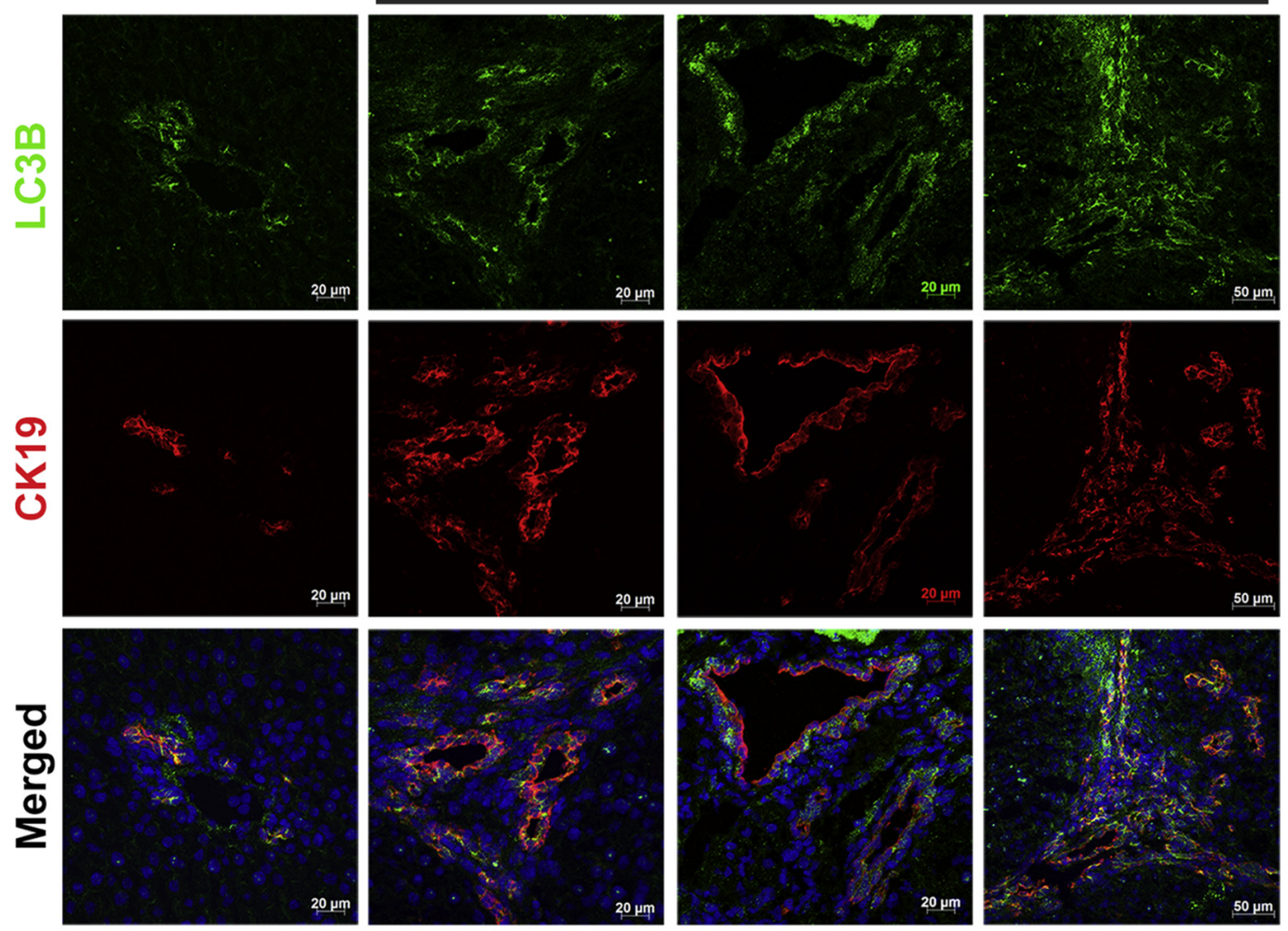

E

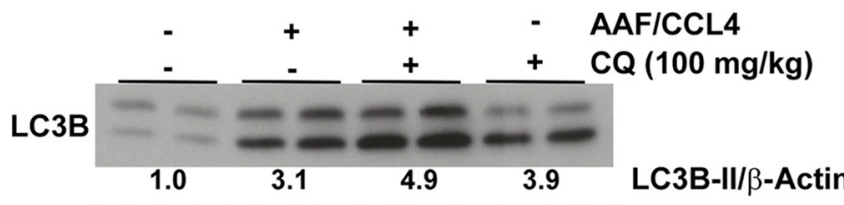

p62

$$
\begin{array}{lllll}
1.0 & 1.5 & 2.0 & 1.4 & \text { p62/ } \beta \text {-Actin }
\end{array}
$$

Actin 
in glandular, tubular structures that lack clear lumens (Figure 3); these structures were similar to the characteristics of DR. ${ }^{12}$

CK19 is a well-known marker for bile ductular lineage and can label the process of DR. ${ }^{25}$ Therefore, double staining with LC3B and CK19 was performed to determine whether the cells were positive for both markers. LC3B coexpressed with CK19 in linear, circular, and tubular structures, and the pattern of LC3B and CK19 staining had good consistency (Figure 4A).

In the next step, transmission electron microscopy was used to detect autophagy features specifically in proliferating bile ductules. Ultrastructurally, several autophagic vacuoles were observed in damaged small bile ducts (composed of four to eight cells) in the cirrhotic liver, whereas little vacuole formation was observed in the normal liver (Figure 4, B-D). Intact mitochondria can be observed in the cytoplasm of control cells; however, in cirrhotic cells, degradative autophagic vacuoles, which usually have only one limiting membrane and contain electron-dense amorphous material, were observed in the cytoplasm (Figure 4, B-D). These data demonstrated that bile ductular cells in cirrhosis sections clearly showed autophagic response elevation.

Several studies indicate that the extent of DR closely correlates with the severity of fibrosis. ${ }^{13-17}$ Hence, we next compared the correlation between autophagy and the status of fibrosis. In noncirrhotic livers, LC3B plus CK19 dual staining was primarily expressed in the mature bile ducts (Figure 5, A and B), whereas in cirrhotic livers, reaction products localized in the DR of portal areas, including bile duct, ductule structures, and cell clusters (Figure 5C). Moreover, a progressive increase in LC3B and CK19 expression was observed as the severity of fibrosis increased from the F1 stage to the F2 and F4 stages (Figure 5); the correlation between the expression levels of LC3B and CK19 was statistically significant $(P<0.001)$ (Figure 5E).

\section{Correlation between Increased Autophagy Marker and DR}

In addition to CHC-related cirrhosis, other types of cirrhosis, including chronic hepatitis B, alcoholic liver disease, and primary biliary cirrhosis, were also assessed whether a similar pattern of protein expression would be seen across a spectrum of liver disease. LC3B and CK19 colocalization was associated with bile ductular proliferation in all other liver diseases (Figure 6).

Similar CK19-positive DR has also been described in the rodent model $^{18}$; we, therefore, studied whether increased DR was accompanied by autophagic changes during the progression of experimental cirrhosis in rat. Sprague-Dawley rats were fed with chronic AAF in combination with $\mathrm{CCL}_{4}$ administration for 6 weeks. At the end of the treatment, the ratio of the liver weight/body weight, serum AST, and ALT significantly increased in $\mathrm{AAF} / \mathrm{CCL}_{4}$ rats compared with untreated rats (Figure 7A). On histological examination, the livers of $\mathrm{AAF} / \mathrm{CCL}_{4}$ rats exhibited marked fibrosis with portalcentral bridging (Figure 7B). In addition, CK19-positive ductular cells extended out into lobules, forming bridges that separate islands of parenchyma, in the livers of $\mathrm{AAF} / \mathrm{CCL}_{4}$ rats (Figure $7 \mathrm{C}$ ), supporting the clinical relevance of this animal model. By using dual-immunofluorescence staining, we observed that the CK19-positive cells were also positive for LC3B staining (Figure 7D), which was similar to that observed in humans.

In addition, we monitored the autophagic flux analyzing LC3B turnover assay using CQ, which can block autophagy degradation in the lysosome, thereby triggering the accumulation of autophagosome. We observed that in the presence of CQ, the LC3B-II levels induced by $\mathrm{AAF} / \mathrm{CCL}_{4}$ were further increased compared with either $\mathrm{AAF} / \mathrm{CCL}_{4}$ or $\mathrm{CQ}$ treatment alone (Figure 7E). Moreover, p62 levels were increased by $\mathrm{CQ}$, suggesting that autophagic flux was increased during fibrogenesis in the $\mathrm{AAF} / \mathrm{CCL}_{4}$ model.

Our data showed that p62 levels did not decrease in the $\mathrm{AAF} / \mathrm{CCL}_{4}$ rats despite autophagic degradation; this finding might be explained by aberrant accumulation of p62 in a detergent-insoluble fraction. ${ }^{23,24}$ Consistently, we observed that a substantial amount of p62 in a Triton X-100insoluble fraction accounts for the increase of $\mathrm{p} 62$ protein in $\mathrm{AAF} / \mathrm{CCL}_{4}$ rats (Supplemental Figure S1). In contrast, $\mathrm{AAF} / \mathrm{CCL}_{4}$-induced degradation of $\mathrm{p} 62$ was observed in a Triton X-100-soluble fraction, and this was rescued when autophagic degradation is inhibited by $\mathrm{CQ}$.

\section{Inhibited Autophagy Alleviated $\mathrm{AAF} / \mathrm{CCL}_{4}$-Induced Liver Fibrosis}

To prove that autophagy is required for progression of DR-fibrosis, we co-administered $\mathrm{CQ}$ in $\mathrm{AAF} / \mathrm{CCL}_{4}$ rats.

\footnotetext{
Figure 7 Increased autophagy marker correlates with ductular reaction in the rats with 2-acetylaminofluorene (AAF)/carbon tetrachloride $\left(\mathrm{CCL}_{4}\right)$ administration. A: After 6 weeks of treatment, rats were sacrificed and the livers were removed to weight. Blood samples were collected to measure serum aspartate aminotransferase (AST) and alanine aminotransferase (ALT) levels by using enzyme-linked immunosorbent assay. B: Formalin-fixed, paraffinembedded liver tissues were divided into sections and followed by Sirius red staining. Extensive fibrous septae that separate hepatocyte into nodules are present in $\mathrm{AAF} / \mathrm{CCL}_{4}$ liver. C: Cytokeratin 19 (CK19) immunofluorescence detection (red) on frozen liver sections. Positive signal was observed in bile duct of normal liver and ductular cells along with bridging fibrous septa of AAF/CCL4 livers. D: Dual-immunofluorescence staining of autophagy marker LC3B (green) combined with CK19 (red). Merged images reveal that LC3B is expressed in the bile duct of normal liver and the ductular cells of AAF/CCL4 livers. E: Evaluation of autophagic flux using lysosomal inhibitor chloroquine (CQ). Normal and AAF/CCL 4 -treated rats were given phosphate-buffered saline or CQ 4 hours before sacrifice. Levels of LC3B-II and p62 quantified by densitometry (normalized to the loading control and to the normal without CQ) are shown below the blot. Representative Western blot analysis is from three independent experiments. Data are expressed as the means \pm SEM from at least three independent experiments (A). $n=3$ rats in each group (A); $n=2$ to 4 rats for each experimental condition $(\mathbf{E}) .{ }^{*} P<0.05$, Student's unpaired $t$-test.
} 
Treatment with $50 \mathrm{mg} / \mathrm{kg}$ per day CQ was initiated when rats were pretreated with $\mathrm{AAF} / \mathrm{CCL}_{4}$ for 2 weeks, followed by 4 weeks of cotreatment.

CQ treatment showed a decline of DR, as evidenced by immunohistochemistry and Western blot analysis for CK19 expression (Figure 8). Moreover, autophagy suppression ameliorated liver injury and decreased fibrosis induced by $\mathrm{AAF} / \mathrm{CCL}_{4}$, as measured by Sirius red (Figure $8 \mathrm{~A}$ ) and by the levels of profibrogenic gene expression (Figure 8B) in the liver.

However, combined $\mathrm{CQ}$ and $\mathrm{AAF} / \mathrm{CCL}_{4}$ treatment did not further increase the endogenous LC3B-II levels (Figure 8C), although the lysosomal activity was suppressed, as evidenced by decreased expression levels and activity of cathepsin D (Supplemental Figure S2). Because our CQ was co-administered with injury in the early stage of liver fibrosis, we hypothesized that the ability of CQ to neutralize inflammation, which is a well-known indication used in a clinic, ${ }^{26}$ might counteract the $\mathrm{AAF} / \mathrm{CCL}_{4}$-induced toxicity and, as a result, hinder the induction of autophagy. The mechanism by which $\mathrm{CQ}$ suppresses $\mathrm{AAF} / \mathrm{CCL}_{4}$ induced fibrosis remains to be further clarified.

\section{Discussion}

The autophagy function in hepatic fibrosis has only been recently described, ${ }^{8,9}$ and whether increased autophagy results in human cirrhosis remains to be investigated. The research conducted herein is directed at understanding the autophagic functioning of the human cirrhotic liver. We demonstrated that autophagy markers were strongly up-regulated (on the basis of LC3B immunostaining, Western blot analysis, and transmission electron microscopy) in cirrhotic tissues compared with noncirrhotic tissues. The increased level of LC3B mRNA in cirrhotic livers further provided correlative data related to the induction of autophagy. We also verified that the increased number of autophagosomes in cirrhotic tissues was caused by an enhanced autophagic flux rather than by impaired autophagosome maturation. First, we verified the occurrence of the late steps of autophagolysosome formation in cirrhotic tissues by monitoring the colocalization of LC3B with LAMP-1. Second, we confirmed an increased lysosomal activity by examining the maturation of cathepsin D. Furthermore, apart from static measurements in patient samples, autophagic flux was also monitored by treating $\mathrm{AAF} / \mathrm{CCL}_{4}$ rats with $\mathrm{CQ}$. Increased levels of LC3B and p62 by $\mathrm{CQ}$ suggested that autophagic flux increased during fibrogenesis in the AAF/ $\mathrm{CCL}_{4}$-treated model.

An increased number of autophagosomes in hepatic $\mathrm{C}$ virus (HCV)-infected cells and/or livers had been described previously. ${ }^{27,28}$ Autophagy was proposed to be nonfunctional in these reports because no decreases in the p62 protein were observed. ${ }^{29}$ However, another study demonstrated that a selective autophagy for lipids rather than a defective process is induced by $\mathrm{HCV}$ infection. ${ }^{30} \mathrm{By}$ using lysosomal inhibitor approaches, the colocalization of
LC3 and LAMP-1 and a tandem fluorescent-tagged LC3 to determine autophagic flux, Vescovo et $\mathrm{al}^{30}$ demonstrated that an HCV-induced autophagic process can advance to completion. It is now evident that autophagosomes can engulf a substrate selectively rather stochastically and that ubiquitinated proteins, ribosomes, mitochondria, peroxisomes, and intracellular bacteria may all be selectively targeted for autophagic degradation. ${ }^{31}$ Therefore, consistent with the previous reports, ${ }^{30}$ our results showed an absence of decreased level in p62 protein and increased autophagic markers in cirrhotic patients with $\mathrm{HCV}$ infection.

To further exclude the possibility that the observed autophagic changes were HCV specific, experiments were repeated by analyzing cirrhotic livers from patients with chronic hepatitis B, alcoholic liver disease, and primary biliary cirrhosis and from a rodent model of $\mathrm{AAF} / \mathrm{CCL}_{4}$. Our dual-immunofluorescence analysis of cirrhotic livers localized LC3B to CK19-positive ductular cells, providing the first in vivo evidence, to our knowledge, that autophagy regulation could occur in other fibrogenic cells apart from HSCs. Consistently, Sasaki et $\mathrm{al}^{32}$ also reported recently that autophagy is frequently seen in bile ductular cells in primary biliary cirrhosis. Although our work fails to demonstrate increased LC3B punctate in $\alpha$-SMA-positive HSCs (Figure 3 and Supplemental Figure S3), it is possible that $\alpha$-SMA-positive HSCs undergoing autophagy activation were not detected in our experimental condition or that $\alpha$-SMA-negative HSCs underwent autophagy activation. Further studies are required to clarify this point by tracking the behavior of both types of HSCs in the liver. Nevertheless, our data showed that CQ treatment, leading to decreased expression of $\alpha$-SMA, supported a role for autophagy in HSC activation (Figure 8, B and C).

There are two mechanisms proposed to demonstrate how DR promotes liver fibrosis. ${ }^{33}$ First, reactive ductular cells can secrete profibrogenic factors, which then activate matrix-producing cells. Numerous studies have demonstrated an intimate cross talk between ductular cells and $\alpha$-SMA-positive myofibroblasts. ${ }^{18,34,35}$ In this regard, our data showed enrichment of $\alpha$-SMA-positive cells in close proximity to the LC3B-positive ductule structures (Supplemental Figure S3), providing evidence that autophagy has a role in this paracrine interaction between DR and myofibroblast. The other mechanism is that cells that are involved in DR during chronic liver injury are capable of epithelial-mesenchymal transition (EMT),${ }^{14,36-38}$ which is a novel mechanism considered to contribute to organ fibrosis. ${ }^{39}$ Svegliati-Baroni et $\mathrm{al}^{14}$ have demonstrated that the nuclear expression of Snail, down-regulation of E-cadherin, and expression of fibroblast-specific protein-1 by CK7-positive cells in HCV liver biopsy specimens provide clear evidence that these ductular cells actively engage in EMT. Rygiel et $\mathrm{al}^{37}$ also documented co-expression of epithelial and mesenchymal markers in DR cells in human tissues. Recently, a study found that starvation-induced autophagy could induce the expression of EMT markers 
A

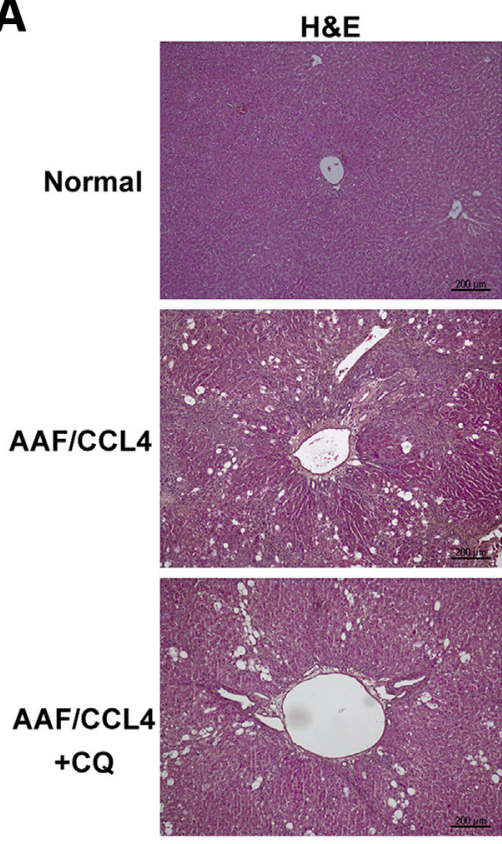

B

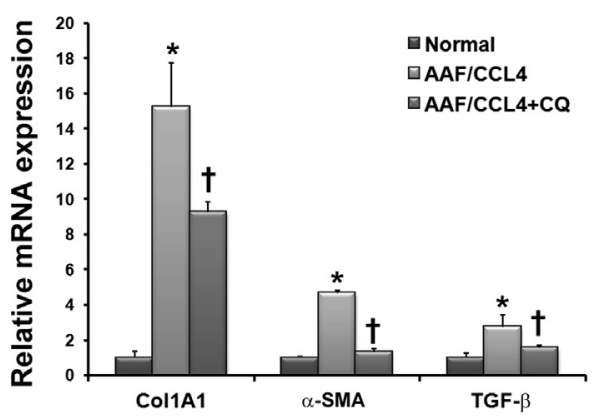

Sirus red
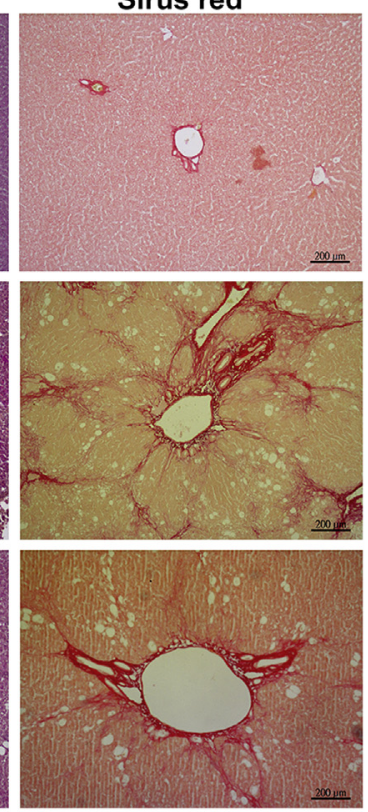
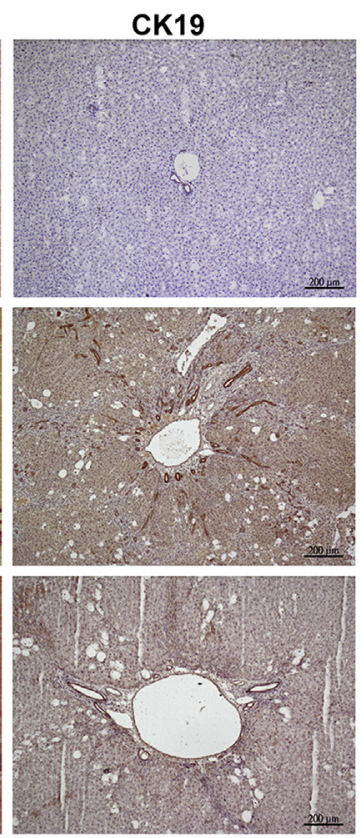
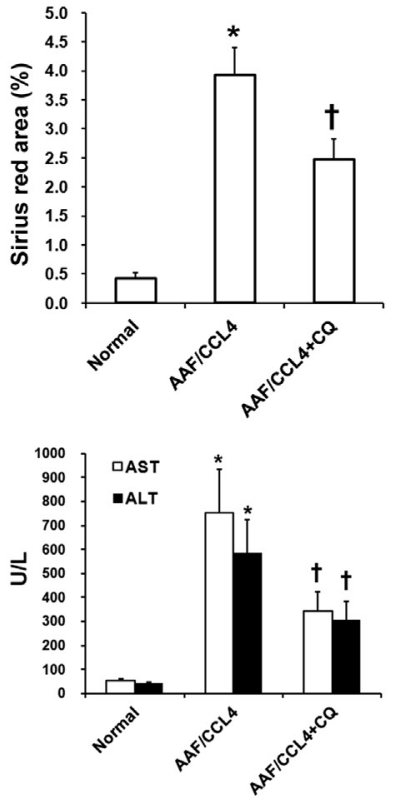

C
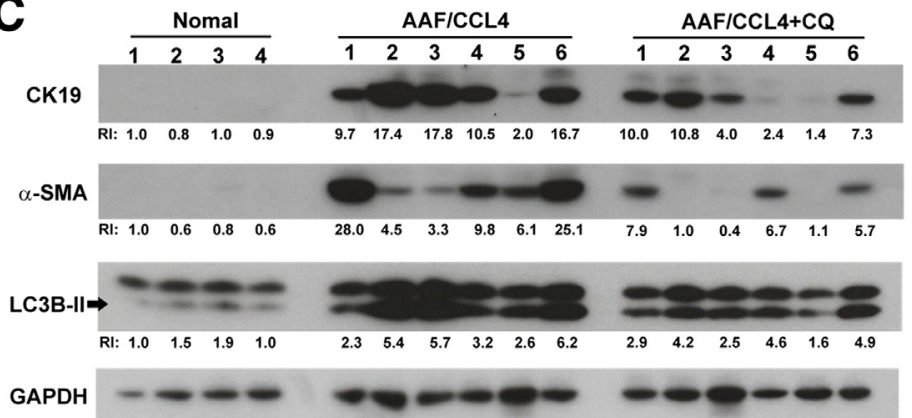

Figure 8 Chloroquine (CQ) treatment attenuates 2-acetylaminofluorene (AAF)/carbon tetrachloride ( $\left.\mathrm{CCL}_{4}\right)$-induced liver fibrosis. Treatment with $50 \mathrm{mg} / \mathrm{kg}$ per day $C Q$ was initiated when rats were pretreated with $A A F / C C L$ for 2 weeks, followed by 4 weeks of cotreatment. A: Normal, $A A F / C C L_{4}$, and AAF/CCL $+C Q$ liver were stained with hematoxylin and eosin (H\&E), Sirius red, and immunohistochemistry for cytokeratin 19 (CK19). Quantification of areas stained for Sirius red and serum aspartate aminotransferase (AST) and alanine aminotransferase (ALT) levels by using enzyme-linked immunosorbent assay. B: Expression of collagen type $1 \propto 1$ (Col1A1), $\alpha$-smooth muscle actin ( $\alpha$-SMA), and transforming growth factor (TGF)- $\beta 1$ mRNA in liver tissues of normal, AAF/CCL $\mathrm{CCL}_{4}+\mathrm{CQ}$ rats. Statistical analysis was analyzed by Student's unpaired $t$-test. C: Representative Western blot analysis depicts levels of CK19, $\alpha$-SMA, LC3B, and glyceraldehyde-3-phosphate dehydrogenase (GAPDH) in liver tissues of normal, $A A F / C C L_{4}$, and $A A F / C C L_{4}+C Q$ rats. Relative intensity (RI) shown was calculated by normalization of the intensities of each marker to the loading control and to the number 1 of normal group. Each lane represents one rat. Data are means $\pm \operatorname{SEM}(\mathbf{A}$ and $\mathbf{B}) . n=4$ to 6 rats per group (A and $\mathbf{B})$. ${ }^{*} P<0.05$ versus normal group; ${ }^{\dagger} P<0.05$ versus AAF/CCL $\mathrm{Croup}$.

and invasion in hepatic carcinoma cells through a transforming growth factor- $\beta / \mathrm{Smad} 3$ signaling-dependent manner. ${ }^{40}$ Whether activation of autophagy may precede EMT of bile ductular cells in DR remains to be investigated.

Herein, we found that LC3B staining was strongly positive in mature bile ducts in noncirrhotic human livers and normal rat liver (Figure 5, A and B, and Figure 7D), indicating that autophagy is required for normal biliary function. Autophagy can remove oxidatively damaged mitochondria; therefore, it may assist in cholangiocyte survival in damaged livers, where oxidative stress -induced up-regulation of p21 inhibits hepatocyte proliferation. ${ }^{41}$ Selective growth advantage for cholangiocyte may serve as a permissive mechanism for an exaggerated DR. In support of this speculation, our data indicated that autophagy was significantly associated with the expansion of the DR in liver cirrhosis. Thus, this study provides a new mechanism for explaining the preferential emergence of DR in many liver diseases.

In conclusion, we provided the evidence of increased autophagy markers in clinical and $\mathrm{AAF} / \mathrm{CCL}_{4}$-induced experimental cirrhotic livers. We also demonstrated that autophagy is significantly associated with the expansion of DR. Furthermore, to prove cause-effect, we used this AAF/ $\mathrm{CCL}_{4}$ rat model to validate that blocking autophagy by CQ inhibits the progression of DR and liver fibrosis. These findings suggest that pharmacological modulation of autophagy may be a potential therapeutic strategy for the management of liver cirrhosis. 


\section{Acknowledgment}

We thank the imaging core staff at the First Core Labs, National Taiwan University College of Medicine, for their technical assistance.

\section{Supplemental Data}

Supplemental material for this article can be found at http://dx.doi.org/10.1016/j.ajpath.2015.05.010.

\section{References}

1. Yang Z, Klionsky DJ: Eaten alive: a history of macroautophagy. Nat Cell Biol 2010, 12:814-822

2. Choi AM, Ryter SW, Levine B: Autophagy in human health and disease. N Engl J Med 2013, 368:1845-1846

3. Deter RL, Baudhuin P, De Duve C: Participation of lysosomes in cellular autophagy induced in rat liver by glucagon. J Cell Biol 1967, 35:C11-C16

4. Czaja MJ, Ding WX, Donohue TM Jr, Friedman SL, Kim JS, Komatsu M, Lemasters JJ, Lemoine A, Lin JD, Ou JH, Perlmutter DH, Randall G, Ray RB, Tsung A, Yin XM: Functions of autophagy in normal and diseased liver. Autophagy 2013, 9:1131-1158

5. Rautou PE, Mansouri A, Lebrec D, Durand F, Valla D, Moreau R: Autophagy in liver diseases. J Hepatol 2010, 53:1123-1134

6. Ni HM, Williams JA, Yang H, Shi YH, Fan J, Ding WX: Targeting autophagy for the treatment of liver diseases. Pharmacol Res 2012, 66: 463-474

7. Lee UE, Friedman SL: Mechanisms of hepatic fibrogenesis. Best Pract Res Clin Gastroenterol 2011, 25:195-206

8. Hernández-Gea V, Ghiassi-Nejad Z, Rozenfeld R, Gordon R, Fiel MI, Yue Z, Czaja MJ, Friedman SL: Autophagy releases lipid that promotes fibrogenesis by activated hepatic stellate cells in mice and in human tissues. Gastroenterology 2012, 142:938-946

9. Thoen LF, Guimarães EL, Dollé L, Mannaerts I, Najimi M, Sokal E, van Grunsven LA: A role for autophagy during hepatic stellate cell activation. J Hepatol 2011, 55:1353-1360

10. Glaser SS, Gaudio E, Miller T, Alvaro D, Alpini G: Cholangiocyte proliferation and liver fibrosis. Expert Rev Mol Med 2009, 11:e7

11. Gouw ASH, Clouston AD, Theise ND: Ductular reactions in human liver: diversity at the interface. Hepatology 2011, 54:1853-1863

12. Roskams TA, Theise ND, Balabaud C, Bhagat G, Bhathal PS, BioulacSage P, Brunt EM, Crawford JM, Crosby HA, Desmet V, Finegold MJ, Geller SA, Gouw AS, Hytiroglou P, Knisely AS, Kojiro M, Lefkowitch JH, Nakanuma Y, Olynyk JK, Park YN, Portmann B, Saxena R, Scheuer PJ, Strain AJ, Thung SN, Wanless IR, West AB Nomenclature of the finer branches of the biliary tree: canals, ductules, and ductular reactions in human livers. Hepatology 2004, 39: $1739-1745$

13. Clouston AD, Powell EE, Walsh MJ, Richardson MM, Demetris AJ, Jonsson JR: Fibrosis correlates with a ductular reaction in hepatitis C: roles of impaired replication, progenitor cells and steatosis. Hepatology 2005, 41:809-818

14. Svegliati-Baroni G, Faraci G, Fabris L, Saccomanno S, Cadamuro M, Pierantonelli I, Trozzi L, Bugianesi E, Guido M, Strazzabosco M, Benedetti A, Marchesini G: Insulin resistance and necroinflammation drives ductular reaction and epithelial-mesenchymal transition in chronic hepatitis C. Gut 2011, 60:108-115

15. Roskams T, Yang SQ, Koteish A, Durnez A, DeVos R, Huang X, Achten R, Verslype C, Diehl AM: Oxidative stress and oval cell accumulation in mice and humans with alcoholic and nonalcoholic fatty liver disease. Am J Pathol 2003, 163:1301-1311
16. Richardson MM, Jonsson JR, Powell EE, Brunt EM, NeuschwanderTetri BA, Bhathal PS, Dixon JB, Weltman MD, Tilg H, Moschen AR, Purdie DM, Demetris AJ, Clouston AD: Progressive fibrosis in nonalcoholic steatohepatitis: association with altered regeneration and a ductular reaction. Gastroenterology 2007, 133:80-90

17. Wood MJ, Gadd VL, Powell LW, Ramm GA, Clouston AD: Ductular reaction in hereditary hemochromatosis: the link between hepatocyte senescence and fibrosis progression. Hepatology 2014, 59:848-857

18. Chobert MN, Couchie D, Fourcot A, Chobert MN, Couchie D, Fourcot A, Zafrani ES, Laperche Y, Mavier P, Brouillet A: Liver precursor cells increase hepatic fibrosis induced by chronic carbon tetrachloride intoxication in rats. Lab Invest 2012, 92:135-150

19. Haspel J, Shaik RS, Ifedigbo E, Nakahira K, Dolinay T, Englert JA, Choi AM: Characterization of macroautophagic flux in vivo using a leupeptin-based assay. Autophagy 2011, 7:629-642

20. Hung TM, Hu RH, Ho CM, Chiu YL, Lee JL, Jeng YM, Shih DT, Lee PH: Downregulation of alpha-fetoprotein expression by LHX4: a critical role in hepatocarcinogenesis. Carcinogenesis 2011, 32: $1815-1823$

21. Kao YH, Lin YC, Tsai MS, Sun CK, Yuan SS, Chang CY, Jawan B, Lee PH: Involvement of the nuclear high mobility group B1 peptides released from injured hepatocytes in murine hepatic fibrogenesis. Biochim Biophys Acta 2014, 1842:1720-1732

22. Kirkin V, McEwan DG, Novak I, Dikic I: A role for ubiquitin in selective autophagy. Mol Cell 2009, 34:259-269

23. Klionsky DJ, Abdalla FC, Abeliovich H, Abraham RT, AcevedoArozena A, Adeli K, et al: Guidelines for the use and interpretation of assays for monitoring autophagy. Autophagy 2012, 8:445-544

24. Fujita K, Maeda D, Xiao Q, Srinivasula SM: Nrf2-mediated induction of p62 controls Toll-like receptor-4-driven aggresome-like induced structure formation and autophagic degradation. Proc Natl Acad Sci U S A 2011, 108:1427-1432

25. Zhou H, Rogler LE, Teperman L, Morgan G, Rogler CE: Identification of hepatocytic and bile ductular cell lineages and candidate stem cells in bipolar ductular reactions in cirrhotic human liver. Hepatology 2007, 45:716-724

26. Wolf R, Wolf D, Ruocco V: Antimalarials: unapproved uses or indications. Clin Dermatol 2000, 18:17-35

27. Taguwa S, Kambara H, Fujita N, Noda T, Yoshimori T, Koike K, Moriishi K, Matsuura Y: Dysfunction of autophagy participates in vacuole formation and cell death in cells replicating hepatitis $\mathrm{C}$ virus. J Virol 2011, 85:13185-13194

28. Rautou PE, Cazals-Hatem D, Feldmann G, Mansouri A, Grodet A, Barge S, Martinot-Peignoux M, Duces A, Bièche I, Lebrec D, Bedossa P, Paradis V, Marcellin P, Valla D, Asselah T, Moreau R: Changes in autophagic response in patients with chronic hepatitis $\mathrm{C}$ virus infection. Am J Pathol 2011, 178:2708-2715

29. Sir D, Chen WL, Choi J, Wakita T, Yen TS, Ou JH: Induction of incomplete autophagic response by hepatitis $\mathrm{C}$ virus via the unfolded protein response. Hepatology 2008, 48:1054-1061

30. Vescovo T, Romagnoli A, Perdomo AB, Corazzari M, Ciccosanti F, Alonzi T, Nardacci R, Ippolito G, Tripodi M, Garcia-Monzon C, Lo Iacono O, Piacentini M, Fimia GM: Autophagy protects cells from HCV-induced defects in lipid metabolism. Gastroenterology 2012, 142:644-653

31. Yu L, Strandberg L, Lenardo MJ: The selectivity of autophagy and its role in cell death and survival. Autophagy 2008, 4:567-573

32. Sasaki M, Miyakoshi M, Sato Y, Nakanuma Y: Autophagy may precede cellular senescence of bile ductular cells in ductular reaction in primary biliary cirrhosis. Dig Dis Sci 2012, 57:660-666

33. Svegliati-Baroni G, De Minicis S, Marzioni M: Hepatic fibrogenesis in response to chronic liver injury: novel insights on the role of cell-to-cell interaction and transition. Liver Int 2008, 28: $1052-1064$

34. Lorenzini S, Bird TG, Boulter L, Bellamy C, Samuel K, Aucott R, Clayton E, Andreone P, Bernardi M, Golding M, Alison MR, Iredale JP, Forbes SJ: Characterisation of a stereotypical cellular and 
extracellular adult liver progenitor cell niche in rodents and diseased human liver. Gut 2010, 59:645-654

35. Van Hul NK, Abarca-Quinones J, Sempoux C, Horsmans Y, Leclercq IA: Relation between liver progenitor cell expansion and extracellular matrix deposition in a CDE-induced murine model of chronic liver injury. Hepatology 2009, 49:1625-1635

36. Robertson H, Kirby JA, Yip WW, Jones DE, Burt AD: Biliary epithelial-mesenchymal transition in posttransplantation recurrence of primary biliary cirrhosis. Hepatology 2007, 45:977-981

37. Rygiel KA, Robertson H, Marshall HL, Pekalski M, Zhao L, Booth TA, Jones DE, Burt AD, Kirby JA: Epithelial-mesenchymal transition contributes to portal tract fibrogenesis during human chronic liver disease. Lab Invest 2008, 88:112-123

38. Syn WK, Jung Y, Omenetti A, Abdelmalek M, Guy CD, Yang L, Wang J, Witek RP, Fearing CM, Pereira TA, Teaberry V, Choi SS,
Conde-Vancells J, Karaca GF, Diehl AM: Hedgehog-mediated epithelial-to-mesenchymal transition and fibrogenic repair in nonalcoholic fatty liver disease. Gastroenterology 2009, 137:1478-1488

39. Acloque H, Adams MS, Fishwick K, Bronner-Fraser M, Nieto MA: Epithelial-mesenchymal transitions: the importance of changing cell state in development and disease. J Clin Invest 2009, 119: $1438-1449$

40. Li J, Yang B, Zhou Q, Wu Y, Shang D, Guo Y, Song Z, Zheng Q, Xiong J: Autophagy promotes hepatocellular carcinoma cell invasion through activation of epithelial-mesenchymal transition. Carcinogenesis 2013, 34:1343-1351

41. Lunz JG 3rd, Tsuji H, Nozaki I, Murase N, Demetris AJ: An inhibitor of cyclin-dependent kinase, stress-induced p21Waf-1/Cip-1, mediates hepatocyte mito-inhibition during the evolution of cirrhosis. Hepatology $2005,41: 1262-1271$ 